\title{
Radiation damage of amorphous silicon, thin-film, field-effect transistors
}

\author{
J. M. Boudry and L. E. Antonuk \\ Department of Radiation Oncology, University of Michigan, Ann Arbor, Michigan 48109-0010
}

(Received 15 September 1995; accepted for publication 1 January 1996)

The effect of ${ }^{60} \mathrm{Co}$ radiation on the noise and drain-source current characteristics of hydrogenated amorphous silicon ( $a$-Si:H) field-effect transistors (FETs) was examined as a function of dose to cumulative doses as high as $\sim 2 \times 10^{4} \mathrm{~Gy}$. Following these measurements, room-temperature and elevated-temperature annealing of induced radiation damage was examined. The FETs examined are representative of those incorporated in $a$-Si:H arrays under development for various $\mathrm{x}$-ray medical imaging applications. No significant effect upon the noise characteristics of the FETs was observed as a result of the radiation. The predominant drain-source current effect with increasing dose was a shift of the transfer characteristic toward negative gate voltage and/or a decrease of the transfer characteristic subthreshold slope. This resulted in large increases in leakage current for gate voltages where the FETs were initially highly nonconducting. This leakage current increase was less pronounced for more negative gate voltages and was further diminished by maintaining the FETs at a more negative gate voltage during the irradiation. Following the radiation measurements, room-temperature annealing resulted in a $10 \%$ to $50 \%$ reduction in the leakage current in the first day followed by a logarithmic decrease thereafter. Elevated-temperature annealing for $2 \mathrm{~h}$ at $200{ }^{\circ} \mathrm{C}$ restored FET leakage current and threshold voltage properties to their preirradiation values. The irradiation effects are small for cumulative doses less than $\sim 100 \mathrm{~Gy}$, which is larger than the clinical lifetime dose for an imaging detector for chest radiography or for fluoroscopy (with infrequent exposure to the direct beam). For significantly higher dose applications such as mammography, fluoroscopy (with frequent direct beam exposure), and radiotherapy imaging, the results suggest that periodic elevated-temperature annealing or operation of the arrays at more negative gate voltages may be necessary. (c) 1996 American Association of Physicists in Medicine.

Key words: amorphous silicon, field-effect transistor, thin-film transistor, radiation damage

\section{INTRODUCTION}

A promising new technology for real-time, digital $x$-ray imaging has emerged using large-area imaging arrays incorporating thin-film circuity fabricated from hydrogenated amorphous silicon $(a-\mathrm{Si}: \mathrm{H}){ }^{1}$ Generally, the arrays employ pixel designs in which an $a-\mathrm{Si}: \mathrm{H}$ switch is coupled to a sensor. The switch may take the form of a field-effect transistor $(\mathrm{FET}),{ }^{2-7}$ a single diode, ${ }^{8,9}$ or a dual diode combination. ${ }^{10,11}$ Reported operational designs which incorporate an $a$-Si:H FET couple the transistor to an optically sensitive element such as an $a-\mathrm{Si}: \mathrm{H}$ photodiode $\mathrm{e}^{3-5}$ (which provides indirect detection of the radiation by means of an overlying lightemitting converter) or to an amorphous selenium detective layer ${ }^{7}$ which provides direct detection.

Our group has been investigating arrays in which the pixel consists of an $a$-Si:H FET coupled to an $a-\mathrm{Si}: \mathrm{H}$ photodiode sensor. ${ }^{3}$ The FET functions as a switch through manipulation of its gate voltage, $V_{g}$. With the FET nonconducting or "off" ' $\left(V_{g}\right.$ negative, typically -5 to $\left.-10 \mathrm{~V}\right)$, electronhole pairs that are created in the photodiode due to the incident $\mathrm{x}$-ray quanta are integrated in the photodiode capacitance and constitute the imaging signal. In order to sample this stored signal by external electronics, a positive $V_{g}$ is applied $(\sim+5$ to $+10 \mathrm{~V})$ and the FET becomes conducting or "on." An array of such pixels will thus allow for real-time imaging of an $x$-ray intensity profile over a large area. To date, $x$-ray images of anatomical detail have been demonstrated with imagers using arrays as large as $\sim 23 \times 25$ $\mathrm{cm}^{1,12,13}$

A requisite for the use of $a$-Si:H arrays for x-ray imaging is that the sensors and transistors be resistant to radiation damage. Since the array is placed directly in the radiation field, it may be subject to large radiation doses, depending on the application. The largest yearly dose levels expected for medical applications are in radiotherapy imaging, where an array could receive on the order of $10^{4}$ Gy (at an average dose rate of $\sim 50$ to $100 \mathrm{~Gy} /$ day) from the incident, high energy ( $\sim 3$ to $50 \mathrm{MV}$ ) bremsstrahlung photon beam. [For an array $130 \mathrm{~cm}$ from the $\mathrm{x}$-ray source (patient $100 \mathrm{~cm}$ from the source), the estimated yearly dose to the array for the unattenuated beam is approximately: ( 2 to $4 \mathrm{~Gy} /$ patient $) \times(40$ patients/day $) \times(250$ days/year $) \times(100 / 130)^{2}=\sim 1 \times 10^{4}$ to $2 \times 10^{4} \mathrm{~Gy}$.] The value of $10^{4} \mathrm{~Gy}$, therefore, provides a benchmark dose level for which the electrical properties of the $a$-Si:H photodiodes and FETs must not change so as to adversely affect array operation. Previous $x$-ray studies ${ }^{14,15}$ have demonstrated that $a-\mathrm{Si}: \mathrm{H}$ photodiodes exhibit the necessary radiation-damage resistance for such dose levels. This report concentrates on $a$-Si:H FETs and examines the effects of radiation on FET electrical properties that are relevant for array operation.

Both the signal and noise characteristics of $a$-Si:H FETs affect imager performance. (These characteristics also aid in determining radiation damage mechanisms in the FET.) The 
signal characteristics encompass a number of FET properties, all of which are related to the magnitude of the drainsource current, $I_{\mathrm{ds}}$, passing through the FET. These properties include the mobility, $\mu$, the threshold voltage, $V_{T}$, the leakage current, the linearity, and the transfer characteristic. The mobility governs how quickly electrons will pass through a FET and, therefore, influences array readout rate. The threshold voltage determines the gate voltage necessary to switch a transistor into the conducting state. The leakage current, or $I_{\mathrm{ds}}$ for the FET in the nonconducting state, contributes spurious signal and additional noise to the imaging system. The linearity tests the degree to which the FET remains ohmic for certain voltage settings. The transfer characteristic is a measure of $I_{\mathrm{ds}}$ for varying $V_{g}$ and from this measurement, $\mu, V_{T}$, and the leakage current can be obtained.

Previous studies with ionizing radiation have been performed with $\mathrm{x}$ rays ${ }^{16}$ and protons ${ }^{17}$ for $a$-Si:H FETs of similar construction to those examined in the present study. These studies examined several of the aforementioned properties. The first study ${ }^{16}$ examined transfer characteristics for various size FETs after cumulative dose levels of $1 \times 10^{4}$ and $5 \times 10^{4}$ Gy using a ${ }^{60} \mathrm{Co}(\sim 1.25 \mathrm{MeV})$ source. From these transfer measurements, the authors were able to determine $V_{T}$ and transconductance shifts with dose. The measurements did not extend to $V_{g}$ values that would allow for a determination of the effects on the leakage current. The second study ${ }^{17}$ measured $\mu, V_{T}$, noise, and leakage current for a FET as a function of proton fluence (using $1.4 \mathrm{MeV}$ protons) to a total fluence of $10^{14} \mathrm{p} / \mathrm{cm}^{2}$. In general, the results of these two studies indicated that the properties of the FETs in the conducting state were not significantly altered. The results of the second study, ${ }^{17}$ however, suggest that the FETs are significantly affected in the nonconducting state, where the leakage current was found to increase approximately three orders of magnitude after a fluence of $10^{12} \mathrm{p} / \mathrm{cm}^{2}$.

This article presents measurements of the FET noise and drain-source current characteristics as a function of dose using a ${ }^{60} \mathrm{Co}$ source up to a maximum cumulative dose of $\sim 2 \times 10^{4}$ Gy. For the drain-source current characteristics, measurements of $\mu, V_{T}$, leakage current, linearity, and the transfer characteristic are reported. For the noise characteristics, current-noise-power spectra measurements for the FETs in both the conducting and nonconducting mode are presented. In addition, room-temperature and elevatedtemperature annealing of the induced radiation damage are examined. Finally, radiation damage mechanisms and the implications of the measurements for clinical $x$-ray imaging applications are discussed.

\section{MATERIALS AND METHODS}

\section{A. FET description}

While the structure and operation of the FETs used in this study have been previously reported ${ }^{18}$ a short description containing additional pertinent details follows.

The structure of a FET is illustrated schematically in Fig. 1. The metal gate layer is $\sim 80$ to $120 \mathrm{~nm}$ thick, and the

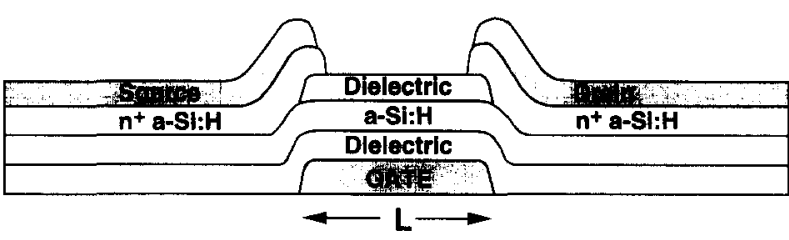

FIG. 1. Cross-sectional drawing (not to scale) showing the structure of the $a$-Si:H transistors used for this study. The length, $L(\sim 15 \mu \mathrm{m})$, is indicated in the figure. (The width, $W(\sim 60 \mu \mathrm{m})$, extends into the page.) The thicknesses of the various layers are listed in the text.

thickness of the source and the drain contacts (also metal) ranges from $\sim 50$ to $500 \mathrm{~nm}$. The dielectric layers consist of amorphous silicon nitride $\left(a-\mathrm{Si}_{3} \mathrm{~N}_{4}: \mathrm{H}\right)$. The "bottom" dielectric layer (the layer directly overlying the gate) is $\sim 300$ $\mathrm{nm}$ thick and the "top" dielectric layer is $\sim 150 \mathrm{~nm}$. The undoped and $n^{+}$doped $a$-Si:H layers have thicknesses of $\sim 30$ to $50 \mathrm{~nm}$ and 50 to $100 \mathrm{~nm}$, respectively. The width, $W$, is $\sim 60 \mu \mathrm{m}$ and the length, $L$, is $\sim 15 \mu \mathrm{m}$ for each of the FETs. An overlying polyimide passivation layer $(\sim 500$ to $2000 \mathrm{~nm}$ thick $)$ and a metal "light shield" ( $\sim 1 \mu \mathrm{m}$ thick $)$, which is placed on top of the passivation layer and covers the basic FET structure, are not shown. The light shield is biased at the same potential voltage as the gate contact. The FETs studied were individual test devices fabricated on the periphery of array substrates containing small $64 \times 40$ pixel format arrays. While these FETs were produced on a four inch wafer processing line using quartz substrates (as opposed to the large-area processing line and glass substrates used for largearea arrays), the basic FET structure is the same as for largearea array FETs.

A brief description of how the FET operates is as follows. (More detailed descriptions of the operation and physics of $a$-Si:H FETs are reported elsewhere. ${ }^{19-21}$ ) For positive $V_{g}$, a mobile electron layer is induced in the $a-\mathrm{Si}: \mathrm{H}$ layer, allowing for a relatively highly conductive path between the drain and source contacts. The current flow between the drain and source, $I_{\mathrm{ds}}$, will then be proportional to the potential difference between the drain and source contacts, known as the drain-source voltage, $V_{\mathrm{ds}}$. [For each measurement, one contact (arbitrarily labeled as the "source") was held at ground $(0 \mathrm{~V})$ potential, and the $V_{g}$ and $V_{\mathrm{ds}}$ potentials were referenced to this ground.] For typical $V_{\mathrm{ds}}(\sim-4$ to $4 \mathrm{~V})$ and positive $V_{g}(\sim 5$ to $10 \mathrm{~V})$ encountered during operation of $a-\mathrm{Si}: \mathrm{H}$ imaging arrays, $I_{\mathrm{ds}}$ is of order $1 \mu \mathrm{A}$. For negative $V_{g}$, holes are induced in the $a-\mathrm{Si}: \mathrm{H}$ layer. Conduction of these holes is minimal because of their low mobility in $a-\mathrm{Si}: \mathrm{H}$ and the blocking $n^{+}$doped layers. For $V_{g}$ sufficiently negative $(\sim-3$ to $-10 \mathrm{~V})$ and less than $V_{\mathrm{ds}}$ by $\sim 1 \mathrm{~V}$ or more, $I_{\mathrm{ds}}$ is typically less than $\sim 0.1 \mathrm{pA}$. In this regime, $I_{\mathrm{ds}}$ is commonly referred to as the leakage current.

The transfer characteristic shown in Fig. 2 illustrates the leakage current and conducting regions as well as the transitional subthreshold region. In the conducting region, it is observed that $I_{\mathrm{ds}}$ increases in a linear fashion with $V_{g}$. A linear fit to the data (as shown in Fig. 2) enables the determination of $\mu$ and $V_{T}$ using the following expression ${ }^{22}$ developed for crystalline silicon FETs: 


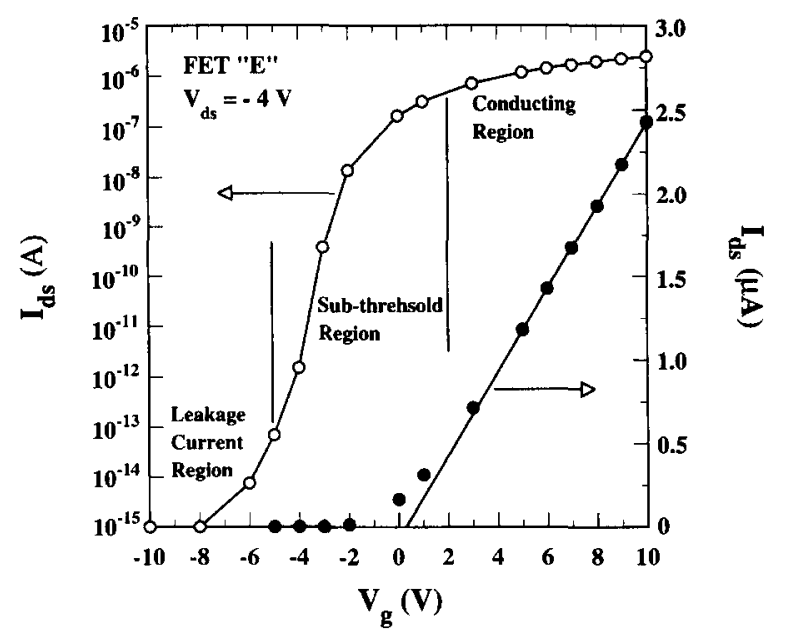

FIG. 2. Transfer characteristic for a 4:1 FET (FET E) measured prior to irradiation at $V_{\mathrm{ds}}=-4 \mathrm{~V}$. The data is plotted vs both a logarithmic (open circles) and a linear (closed circles) vertical axis. The linear fit to the data gives a mobility of $\sim 0.8 \mathrm{~cm}^{2} / \mathrm{Vs}$ and a threshold voltage of $\sim 2.3 \mathrm{~V}$. The error introduced by the measurement system is approximately $\pm 3 \mathrm{fA}$. $I_{\mathrm{ds}}$ values reported as $1 \mathrm{fA}$, which is the smallest current step resolved by the picoammeter, represent measurements for which the current due to the FET could not be differentiated from other external sources of leakage current.

$$
I_{\mathrm{ds}}=\mu \Gamma(W / L) V_{\mathrm{ds}}\left(V_{g}-V_{T}-V_{\mathrm{ds}} / 2\right) .
$$

In this expression, $\Gamma$ is the capacitance per unit area formed between the gate contact and the $a$-Si:H layer $\left(\sim 2 \times 10^{-8}\right.$ $\left.\mathrm{F} / \mathrm{cm}^{2}\right)$ and $W / L$ is the FET aspect ratio. For $V_{\mathrm{ds}} \geqslant V_{g}-V_{T}$, $V_{\mathrm{ds}}$ is set equal to $V_{g}-V_{T}$ in Eq. (1). In general, Eq. (1) is valid for the FET in the conducting mode $\left(V_{g}>V_{T}\right)$ and agreed well with $I_{\mathrm{ds}}$ measurements for the FETs carried out prior to the irradiations. (For $5 \leqslant V_{g} \leqslant 10 \mathrm{~V}$ and $-4 \leqslant V_{\mathrm{ds}} \leqslant 10$ $\mathrm{V}$, the agreement was $\pm 15 \%$.)

\section{B. Radiation damage and annealing measurements}

Two independent studies were performed. Each study examined a particular set of properties of a different group of FETs operated under various conditions. The first part of each study examined the response of various FET electrical properties to radiation dose, and the second part investigated the annealing behavior of these properties. For the purposes of notation, the radiation response measurements of the two studies will be referred to as irradiation \#1 and irradiation \#2 and the annealing measurements for each study will be referenced to the associated irradiations. For both irradiation \#1 (+annealing) and irradiation \#2 (+ annealing), the electrical properties of three individual FETs were examined. The FETs are identified as $\mathrm{A}, \mathrm{B}$, and $\mathrm{C}$ for irradiation \#1 (+annealing) and $D, E$, and $F$ for irradiation \#2 (+annealing), where each letter denotes a separate, individual FET.

A ${ }^{60} \mathrm{Co}$ source was used for the irradiations. For the duration of each irradiation, the FETs were housed in a lighttight, low humidity (relative humidity less than $\sim 5 \%$ ), portable mount. Electrical contact to the FETs was made by wirebonding to a printed circuit board placed in the mount, and measurements of $I_{\mathrm{ds}}$ were performed using a picoammeter (Hewlett-Packard 4140B). Leakage currents from the printed circuit board were subtracted in order to determine the current due to the FET, which introduced a measurement error of $\sim 3 \mathrm{fA}$. A $\sim 0.5 \mathrm{~cm}$ sheet of polystyrene was placed directly over the irradiated FETs to provide an overlying layer of dose buildup material. Through measurements made with a calibrated ion chamber and electrometer and using standard dosimetric techniques, ${ }^{23}$ the dose rate was determined to be $\sim 290$ and $260 \mathrm{~Gy} / \mathrm{h}$ ( $\sim 5 \%$ accuracy) for irradiations \#1 and \#2, respectively.

For irradiation \#1, the following properties for each of the FETs were investigated as a function of radiation dose: (i) mobility, (ii) threshold voltage, (iii) spectral noise for positive $V_{g}$, and (iv) leakage current. Following irradiation \#1, the effect of room-temperature annealing on $\mu, V_{T}$, and leakage current was monitored for 54 days after which the effect of elevated-temperature $\left(\sim 45\right.$ and $\left.180^{\circ} \mathrm{C}\right)$ anneals on these properties was examined. (Room-temperature annealing studies were carried out for extended periods of time after the irradiation in order to allow an unambiguous examination of any annealing effects which would otherwise be very difficult to isolate during the irradiation.) Prior to and after irradiation \#1 (but before the elevated-temperature anneals), the spectral noise for negative $V_{g}$ was measured.

For irradiation \#2, the following quantities were examined as a function of dose: (i) the transfer characteristic (from which $\mu, V_{T}$, and the leakage current are obtained) and (ii) the "linearity" of $I_{\mathrm{ds}}$, which measured the degree to which $I_{\mathrm{ds}}$ obeyed Eq. (1) for $V_{\mathrm{ds}} \ll V_{g}-V_{T}$. [For such voltage settings, Eq. (1) predicts that $I_{\mathrm{ds}}$ will depend on $V_{\mathrm{ds}}$ in a near linear fashion.] Room-temperature annealing behavior was investigated by measuring the transfer characteristic and linearity of the FETs for up to 50 days following irradiation \#2. The effect of an elevated-temperature $\left(\sim 200^{\circ} \mathrm{C}\right)$ anneal on these properties was subsequently investigated. Spectral noise (for positive $V_{g}$ ) measurements were also carried out prior to irradiation $\# 2$ and during the room-temperature annealing interval following the irradiation.

Measurements of the FET electrical properties as a function of dose were carried out between consecutive irradiation "intervals." (An irradiation interval is defined as the time during which the FETs are exposed to the ${ }^{60} \mathrm{Co}$ source.) This ensured that the $I_{\mathrm{ds}}$ values contained no contribution from electron-hole pairs generated by the radiation and, thus, provided an unambiguous determination of the FET electrical properties. Typically, the measurements between the irradiation intervals were completed in 12 to $16 \mathrm{~h}$. For irradiation $\# 1$, the electrical properties were measured after cumulative dose levels of 200, 500, 1000, and $2000 \mathrm{~Gy}$. Thereafter, measurements were carried out between consecutive $2000 \mathrm{~Gy}$ intervals to a total cumulative dose of $2 \times 10^{4} \mathrm{~Gy}$, taking $\sim 12$ days to complete. For irradiation \#2, measurements were performed after similar dose steps up to $1 \times 10^{4} \mathrm{~Gy}$, with the addition of a measurement after $100 \mathrm{~Gy}$. This was completed in $\sim 7$ days.

For irradiations \#1 and \#2, the FETs were biased during the various irradiation intervals. For each FET, a negative $V_{g}$ 
TABLE I. Bias settings applied to the FETs during the irradiation intervals FETs A, B, and C were used for irradiation \#1 (+ annealing) and FETs D, E, and $\mathbf{F}$ for irradiation \#2 (+annealing).

\begin{tabular}{lccc}
\hline \hline FET label & $V_{g}$ setting during & \multicolumn{2}{c}{$V_{\mathrm{ds}}$ setting during } \\
& irradiation intervals (V) & irradiation intervals (V) & Control FET \\
\hline A & -5 & -4 & yes \\
B & -5 & 0 & yes \\
C & -5 & 4 & yes \\
D & -5 & -4 & no \\
E & -10 & -4 & yes \\
F & -10 & 0 & no \\
\hline \hline
\end{tabular}

was applied, reflecting the fact that $V_{g}$ will be negative for over $99 \%$ of the time during normal array operation. For irradiation \#1, a $V_{g}$ value of $-5 \mathrm{~V}$ was used for all FETs as this was the typical value used in previous array studies by our group. As a result of the observations made in this initial study, $V_{g}$ values of -5 and $-10 \mathrm{~V}$ were used in irradiation \#2. The $V_{\mathrm{ds}}$ values for irradiation \#1 correspond to the anticipated range of voltages generated from charge integrating in a photodiode for both $n-i-p\left(-4 \leqslant V_{\mathrm{ds}} \leqslant 0 \mathrm{~V}\right)$ and $p-i-n$ $\left(0 \leqslant V_{\mathrm{ds}} \leqslant 4 \mathrm{~V}\right)$ arrays. (The order ofthe $n$-doped, intrinsic, and $p$-doped layers corresponds to the order of deposition.) For irradiation \#2, $V_{\mathrm{ds}}$ values anticipated for $n-i-p$ photodiode arrays and more negative $V_{g}$ were investigated. For comparison purposes, one bias setting was the same for both irradiations. The $V_{g}$ and $V_{\mathrm{ds}}$ bias settings applied during the irradiation intervals are summarized in Table $I$.

In addition to the irradiated FETs, there were several control FETs. During irradiations \#1 and \#2, the control FETs were treated identically to the irradiated FETs but were not irradiated, thus aiding in determining the significance of any nonradiation effects, such as bias-voltage stress. ${ }^{19-21,24,25}$ For irradiation \#1, three control FETs were used, each biased differently and at settings corresponding to the those shown in Table I for FETs A, B, and C. For irradiation \#2, one control FET was used and was biased in the same fashion as FET E.

Following both irradiations, annealing studies were carried out. For the examination of both room and elevatedtemperature annealing, the FETs were not electrically connected between measurements of the various electrical properties. The FETs remained in the light-tight, lowhumidity mount throughout the room-temperature and $45^{\circ} \mathrm{C}$ annealing investigations. For the examination of elevatedtemperature anneals at $\sim 180^{\circ} \mathrm{C}$ and $200^{\circ} \mathrm{C}$, the FETs were necessarily removed from the portable mount, but were treated so as to receive insignificant exposure to light and ambient humidity levels. Measurements of the control FETs continued for both the room-temperature and elevatedtemperature annealing investigations. For such measurements, the control FETs were treated the same as the irradiated FETs except that they were maintained at room temperature.

\section{Techniques for measurement of FET electrical properties}

The electrical properties of the FETs that were measured as a function of radiation dose and during the annealing studies can be broadly divided into two categories. The first such category of measurements is the drain-source current characteristics, which involved the measurement of $I_{\mathrm{ds}}$ for a variety of $V_{g}$ and $V_{\mathrm{ds}}$ settings. From such measurements, the mobility, threshold voltage, leakage current, transfer characteristic, and linearity of the FETs are obtained. The second category of measurements is the noise characteristics. The detailed experimental technique employed for these two categories of measurements is described below and is further summarized in Table II.

\section{Drain-source current characteristics}

a. $V_{g}$ and $V_{d s}$ settings. For the $\mu$ and $V_{T}$ measurements, $I_{\mathrm{ds}}$ was measured for $5 \mathrm{~V} \leqslant V_{g} \leqslant 10 \mathrm{~V}$ and $V_{\mathrm{ds}}$ equal to $+1 \mathrm{~V}$ (irradiation \#1 and annealing) or $V_{\mathrm{ds}}$ ranging from -4 to +1 $V$ (irradiation \#2 and annealing). Using Eq. (1), $\mu$ and $V_{T}$ values were determined from fits to the data. The leakage current was measured for $V_{g}=-5 \mathrm{~V}$ and $V_{\mathrm{ds}}$ ranging from -4 to $+4 \mathrm{~V}$ for irradiation \#1 (+annealing). For irradiation $\# 2$ and the associated annealing studies, the transfer characteristic measurements were carried out for $-10 \leqslant V_{g} \leqslant+10 \mathrm{~V}$ and $V_{\mathrm{ds}}=-2$ and $-4 \mathrm{~V}$, and the FET linearity was examined for $V_{g}=+10 \mathrm{~V}$ and $-2 \leqslant V_{\mathrm{ds}} \leqslant+2 \mathrm{~V}$.

b. Transient behavior. For the various $V_{g}$ and $V_{\mathrm{ds}}$ settings, $I_{\mathrm{ds}}$ was measured for a given temporal interval following application of the bias settings. The length of the temporal interval, $T_{i}$, varied for the different voltages, and, prior to the irradiations, $T_{i}$ values were chosen so that $I_{\mathrm{ds}}$ was representative of a steady-state value at the end of the $T_{i}$ interval. As the cumulative dose level to the FETs increased, it was found that, for some voltage settings, the initial $T_{i}$ values were no longer adequate for allowing $I_{\mathrm{ds}}$ to settle. Instead, an increasing component of $I_{\mathrm{ds}}$ was observed so that the $I_{\mathrm{ds}}$ value determined at $T_{i}$ was not representative of a steadystate value. This behavior is shown in Fig. 3 for FET E. For the initial $(0 \mathrm{~Gy})$ measurement, $I_{\mathrm{ds}}$ is observed to reach a steady-state value well within the initial 3 min interval. For the $8 \mathrm{kGy}$ measurement, the behavior of $I_{\mathrm{ds}}$ after $3 \mathrm{~min}$ is not indicative of steady-state behavior. Eventually, after an approximately $10 \mathrm{~min}$ interval, $I_{\mathrm{ds}}$ shows steady-state behavior.

For the voltage settings and dose levels for which $I_{\mathrm{ds}}$ displayed behavior similar to that seen for the $8 \mathrm{kGy}$ data in Fig. 3, the average value of $I_{\mathrm{ds}}$ for these settings is represented in the data plots as follows. The data point denotes the value of $I_{\mathrm{ds}}$ at the initial $T_{i}$ value. A single-sided error bar, extending to larger $I_{\mathrm{ds}}$ values, is representative of the range of values covered by the increasing component of $I_{\mathrm{ds}}$ before reaching a steady-state value. The single-sided error bar was either determined by measurement of $I_{\mathrm{ds}}$ for longer times (up to $30 \mathrm{~min}$ ) after bias application or was deduced from subsequent analysis of the transient behavior.

Initial $T_{i}$ values for the $I_{\mathrm{ds}}$ measurements were $15 \mathrm{~s}, 2$ $\min$, and $3 \min$ for $V_{g}>0 \mathrm{~V}, V_{\mathrm{ds}} \leqslant V_{g} \leqslant 0$, and $V_{g} \leqslant V_{\mathrm{ds}}$, respectively. These $T_{i}$ values remained the same throughout 
TABLE II. Summary of the voltage settings, $T_{i}$ values, and frequency ranges used for the measurements of the drain-source current and spectral noise characteristics. (The settings also apply for the annealing measurements associated with each irradiation.) For irradiation \#2 (+annealing), $\mu, V_{T}$, and the leakage current were obtained from the transfer characteristic measurements with the exception of the $\mu$ and $V_{T}$ measurements for $V_{\mathrm{ds}}=1 \mathrm{~V}$, which were performed separately.

\begin{tabular}{|c|c|c|c|c|}
\hline Measurement & $V_{g}$ settings $(\mathrm{V})$ & $V_{\mathrm{ds}}$ settings $(\mathrm{V})$ & $T_{i}$ values $(\mathrm{s})$ & Frequency $(\mathrm{Hz})$ \\
\hline \multicolumn{5}{|l|}{ Irradiation \#1 } \\
\hline Mobility, $\mu$ & $5 \leqslant V_{g} \leqslant 10$ & 1 & 15 & $\cdots$ \\
\hline Threshold voltage, $V_{T}$ & $5 \leqslant V_{g}^{8} \leqslant 10$ & 1 & 15 & $\cdots$ \\
\hline Leakage current & $-5^{8}$ & $-4 \leqslant V_{\mathrm{ds}} \leqslant 4$ & 180 & $\cdots$ \\
\hline \multicolumn{5}{|l|}{ Spectral noise $\left(+V_{g}\right)$} \\
\hline Flicker & 9 & $-4,4$ & $\ldots$ & $0.08 \leqslant f \leqslant 10$ \\
\hline Thermal & 9 & 0 & & $10^{2} \leqslant f \leqslant 10^{3}$ \\
\hline \multicolumn{5}{|l|}{ Spectral noise $\left(-V_{g}\right)$} \\
\hline Preirradiation & -5 & $-6.5 \leqslant V_{\mathrm{ds}} \leqslant-5.5$ & $\ldots$ & $0.08 \leqslant f \leqslant 10$ \\
\hline Postirradiation & -5 & $-6.5 \leqslant V_{\mathrm{ds}} \leqslant-2.0$ & & $0.08 \leqslant f \leqslant 10$ \\
\hline \multicolumn{5}{|l|}{ Irradiation \#2 } \\
\hline Mobility, $\mu$ & $5 \leqslant V_{g} \leqslant 10$ & $-4,-2,1$ & 15 & $\cdots$ \\
\hline Threshold voltage, $V_{T}$ & $5 \leqslant V_{g} \leqslant 10$ & $-4,-2,1$ & 15 & $\cdots$ \\
\hline Leakage current & $-10 \leqslant V_{g}<V_{\mathrm{ds}}$ & $-4,-2$ & 180 & $\cdots$ \\
\hline Transfer characteristic & $-10 \leqslant V_{g} \leqslant 10$ & $-4,-2$ & $\begin{array}{l}15\left(V_{g}>0 V\right) \\
120\left(V_{\mathrm{ds}} \leqslant V_{g} \leqslant 0 V\right) \\
180\left(V_{g}<V_{\mathrm{ds}}\right)\end{array}$ & $\cdots$ \\
\hline Linearity & 10 & $-2 \leqslant V_{\mathrm{ds}} \leqslant 2$ & 15 & $\cdots$ \\
\hline \multicolumn{5}{|l|}{ Spectral noise $\left(+V_{g}\right)$} \\
\hline Flicker & 9 & $-4,4$ & & $0.08 \leqslant f \leqslant 10$ \\
\hline Thermal & 9 & 0 & $\cdots$ & $10^{2} \leqslant f \leqslant 10^{3}$ \\
\hline
\end{tabular}

the two irradiations (and subsequent annealing studies) except for $V_{g}$ values less than $V_{\mathrm{ds}}$ by approximately $4 \mathrm{~V}$ (and greater) and various cumulative dose levels. For these settings, longer $T_{i}$ values were necessary and the $I_{\mathrm{ds}}$ values are represented as discussed above.

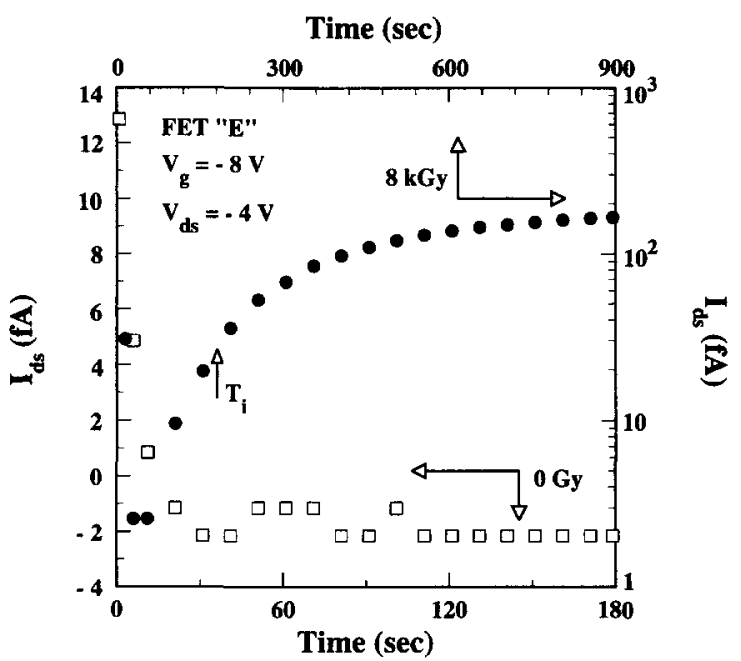

Fig. 3. $I_{\mathrm{ds}}$ transient behaviors for cumulative doses of $0 \mathrm{~Gy}$ (open squares) and $8 \mathrm{kGy}$ (closed circles). The $V_{g}$ and $V_{\mathrm{ds}}$ settings applied for the measurement of $I_{\mathrm{ds}}$ are indicated in the figure. (For the remaining figures, the bias settings applied for the measurement of $I_{\mathrm{ds}}$, rather than the bias settings applied during the irradiation intervals, are indicated.) The bottom and left axes refer to the $0 \mathrm{~Gy}$ data and the top and right axes to the $8 \mathrm{kGy}$ data. Initially ( $0 \mathrm{~Gy})$, a $T_{i}$ value of $3 \mathrm{~min}$ was more than sufficient for determining a steady-state value while after $8 \mathrm{kGy}$, this $T_{i}$ value is no longer adequate (as indicated by the arrow labeled $T_{i}$ ) and approximately $10 \mathrm{~min}$ are required for $I_{\mathrm{ds}}$ to settle. The negative values of $I_{\mathrm{ds}}$ for $0 \mathrm{~Gy}$ are a result of PC board leakage currents.

\section{Noise characteristics}

The drain-source current fluctuations were characterized by measuring the current-noise-power spectral density, $S_{I}$, of $I_{\mathrm{ds}}$ for various $V_{g}$ and $V_{\mathrm{d} s}$. (The measurement technique for $S_{I}$ is detailed elsewhere. $\left.{ }^{18}\right)$ For positive $V_{g}\left(V_{g}=9 \mathrm{~V}\right)$, the flicker (or " $1 / f$ "),$S_{F}$, and thermal, $S_{T}$, noise components of $S_{I}$ were measured. Measurements of $S_{F}$ (for $V_{g}=9 \mathrm{~V}$ ) were performed for $V_{\mathrm{ds}}=-4$ and $4 \mathrm{~V}$ and frequencies out to 10 $\mathrm{Hz}$, and $S_{T}$ was measured for $V_{\mathrm{ds}}=0 \mathrm{~V}$ and $10^{2} \leqslant f \leqslant 10^{3} \mathrm{~Hz}$. For negative $V_{g}\left(V_{g}=-5 \mathrm{~V}\right), S_{I}$ measurements out to $10 \mathrm{~Hz}$ were performed. The $V_{\mathrm{ds}}$ settings for the negative $V_{g}$ spectral measurements were chosen so that $I_{\mathrm{ds}}$ was greater than $\sim 0.5$ pA. At such current levels, the noise due to the FET can be resolved from the measurement system noise. Prior to irradiation $\# 1, V_{\mathrm{ds}}$ values for the $S_{I}$ measurements at negative $V_{g}$ ranged from -5.5 to $-6.5 \mathrm{~V}$ and afterward from -2 to $-6.5 \mathrm{~V}$

\section{RESULTS}

\section{A. Radlation damage measurements}

\section{Drain-source current characteristics}

a. Mobility and threshold voltage. The mobility of the FETs was not significantly altered by the radiation. For both irradiations \#1 and \#2, the mobility of the irradiated and control FETs remained in the range of 0.70 to $0.82 \mathrm{~cm}^{2} / \mathrm{Vs}$. The mobility of the irradiated FETs tended to remain stable, or slightly increase with dose, with a maximum increase of $\sim 0.04 \mathrm{~cm}^{2} / \mathrm{Vs}$. The mobility for each of the control FETs remained constant to the level of $\pm 0.01 \mathrm{~cm}^{2} / \mathrm{Vs}$ during both irradiation studies.

Figure 4 shows the threshold voltage behavior as a function of radiation dose. For irradiation $\# 1, V_{T}$ initially de- 


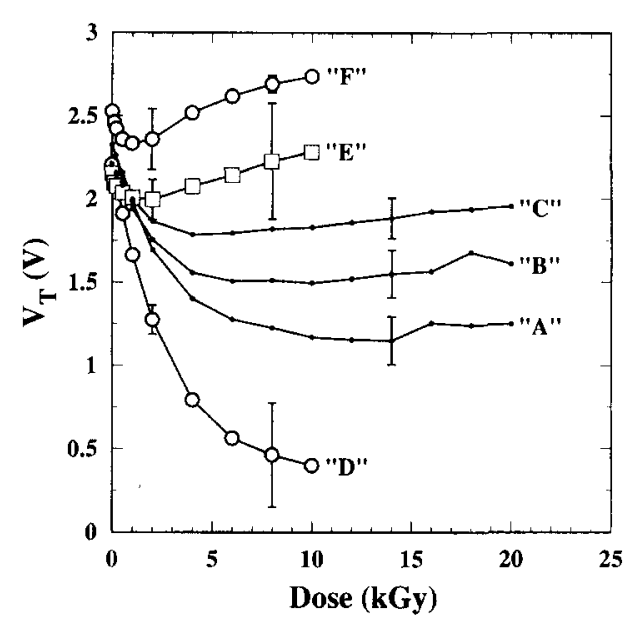

FIG. 4. Threshold voltage behavior as a function of dose for the irradiation \#1 and irradiation \#2 FETs. Representative error bars are shown at a cumulative dose of $14 \mathrm{kGy}$ for FETs $\mathrm{A}, \mathrm{B}$, and $\mathrm{C}$ and at $2 \mathrm{kGy}$ and $8 \mathrm{kGy}$ for FETs D, E, and F.

creased with dose and then remained nearly constant. For irradiation $\# 2, V_{T}$ for FETs $\mathrm{E}$ and $\mathrm{F}$ initially decreased with dose to a cumulative dose of $\sim 1000 \mathrm{~Gy}$ and thereafter slowly increased. FET D monotonically decreased with dose and showed saturation behavior at $\sim 1 \times 10^{4}$ Gy. For FETs $A$ and $\mathrm{D}$, which were biased identically during their respective irradiations, $V_{T}$ is observed to show the largest net shifts. In addition, the general behavior of $V_{T}$ for both FETs is similar in that $V_{T}$ monotonically decreases with dose to a cumulative dose of $\sim 1 \times 10^{4} \mathrm{~Gy}$ and appears to saturate at this dose level. Generally, for each irradiation, the $V_{T}$ shifts were modest for the irradiated FETs, showing a maximum shift of $\sim 1.8 \mathrm{~V}$. As compared to the irradiated FETs, $V_{T}$ values for the controls remained relatively constant, indicating that effects due to factors not related to radiation (such as bias voltage stress) were not significant.

b. Linearity. For irradiation \#2, measurements of $I_{\mathrm{ds}}$ as a function of dose for $V_{g}=+10 \mathrm{~V}$ and $-2 \leqslant V_{\mathrm{ds}} \leqslant 2 \mathrm{~V}$ were compared with those predicted using Eq. (1). Using the $\mu$ and $V_{T}$ values measured for each dose level, agreement between the $I_{\mathrm{ds}}$ measurements and theory was good in this region, typically varying by $4 \%$ for the irradiated and $0.6 \%$ for the control FETs.

c. Leakage current-irradiation \#1. Figure 5 illustrates the leakage current behavior for each of the irradiation \#1 FETs for $V_{g}=-5 \mathrm{~V}$ and various $V_{\mathrm{ds}}$ settings. The general behavior for each $V_{\mathrm{ds}}$ setting is such that $I_{\mathrm{ds}}$ initially increases exponentially with dose before flattening at $\sim 1 \times 10^{4}$ Gy. The magnitude of the net leakage current increase over the entire irradiation varied for the different FETs, indicating that the degree to which the leakage current increases with dose depends upon the $V_{\mathrm{ds}}$ value applied to the FET while exposed to the radiation source. From the figure, it is apparent that the largest change in $I_{\mathrm{ds}}$ has occurred for FET A, followed by FETs $B$ and $C$, respectively. In general, for $V_{\mathrm{ds}} \leqslant-2 \mathrm{~V}$, the asymptotic leakage current values for FET A

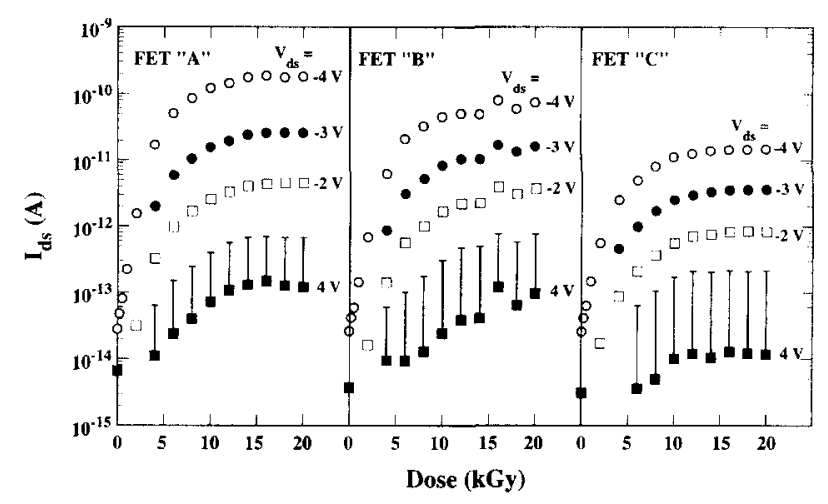

FIG. 5. $I_{\mathrm{ds}}$ vs dose for the three irradiation \#1 FETs. For each $I_{\mathrm{ds}}$ measurement, $V_{g}=-5 \mathrm{~V}$.

were approximately 1.5 to 3 and 5 to 10 times greater than those for FETs $B$ and $C$, respectively. For $V_{d s}=4 \mathrm{~V}$, the asymptotic values of FETs $\mathrm{A}$ and $\mathrm{B}$ were nearly equal and a factor of -3 larger than FET $C$ values.

Measurements of $I_{\mathrm{ds}}$ for $V_{\mathrm{g}}=-5 \mathrm{~V}$ and $V_{\mathrm{ds}}=2 \mathrm{~V}$ were also performed for each of the irradiation \#1 FETs following cumulative doses of $0.2,1.0$, and $2.0 \times 10^{4} \mathrm{~Gy}$. The $I_{\mathrm{ds}}$ values were nearly the same as those at $V_{g}=-5 \mathrm{~V}$ and $V_{\mathrm{ds}}=4 \mathrm{~V}$.

d. Transfer characteristics-irradiation \#2. The results of the leakage current measurements from irradiation \#1 motivated further study into the behavior of the leakage current for more negative $V_{g}$. As shown in the Fig. 2 transfer characteristic for $V_{\mathrm{ds}}=-4 \mathrm{~V}, I_{\mathrm{ds}}$ depends strongly on the gate voltage starting at or near $V_{g}=-5 \mathrm{~V}$. As a consequence, small negative shifts in $V_{T}$ can result in large changes in the leakage current in this region (as was observed in irradiation \#1). For more negative $V_{g}$, it was hypothesized that $I_{\mathrm{ds}}$ would be less sensitive to these $V_{T}$ shifts. In order to investigate this, irradiation \#2 measured the transfer characteristic as a function of dose.

The initial (0 Gy) and final $\left(10^{4} \mathrm{~Gy}\right)$ transfer characteristic measurements at $V_{\mathrm{ds}}=-4 \mathrm{~V}$ are shown in Fig. 6(a) for the three irradiated FETs. For each FET, an increase in leakage current and a decrease in the subthreshold slope is evident. For $V_{g} \geqslant 5 \mathrm{~V}, I_{\mathrm{ds}}$ is virtually unaffected by the radiation. The transfer data for FETs E and F are very similar, while the largest changes in leakage current due to the irradiation occured for FET D. This suggests that the degree of radiation damage is dependent on the $V_{g}$ value applied during the irradiation and is independent of the $V_{\mathrm{ds}}$ value for $V_{g}=-10$ $V$. Figure 6(b) illustrates the response of the leakage current to radiation dose for the three FETs. The response is similar for the different $V_{g}$ settings, initially increasing exponentially with dose before saturating at $\sim 10^{4} \mathrm{~Gy}$. The behavior of $I_{\mathrm{ds}}$ for FET D is also observed to be comparable to that of FET A (see Fig. 5), which had the same voltage settings applied during the irradiation.

Transfer characteristic measurements at $V_{\mathrm{ds}}=-2 \mathrm{~V}$ were also performed as a function of dose. In general, for -8 $\leqslant V_{g} \leqslant 1 \mathrm{~V}$, the transfer characteristic measurements at $V_{\mathrm{ds}}=-2 \mathrm{~V}$ were nearly equal to those at $V_{\mathrm{ds}}=-4 \mathrm{~V}$ shifted 

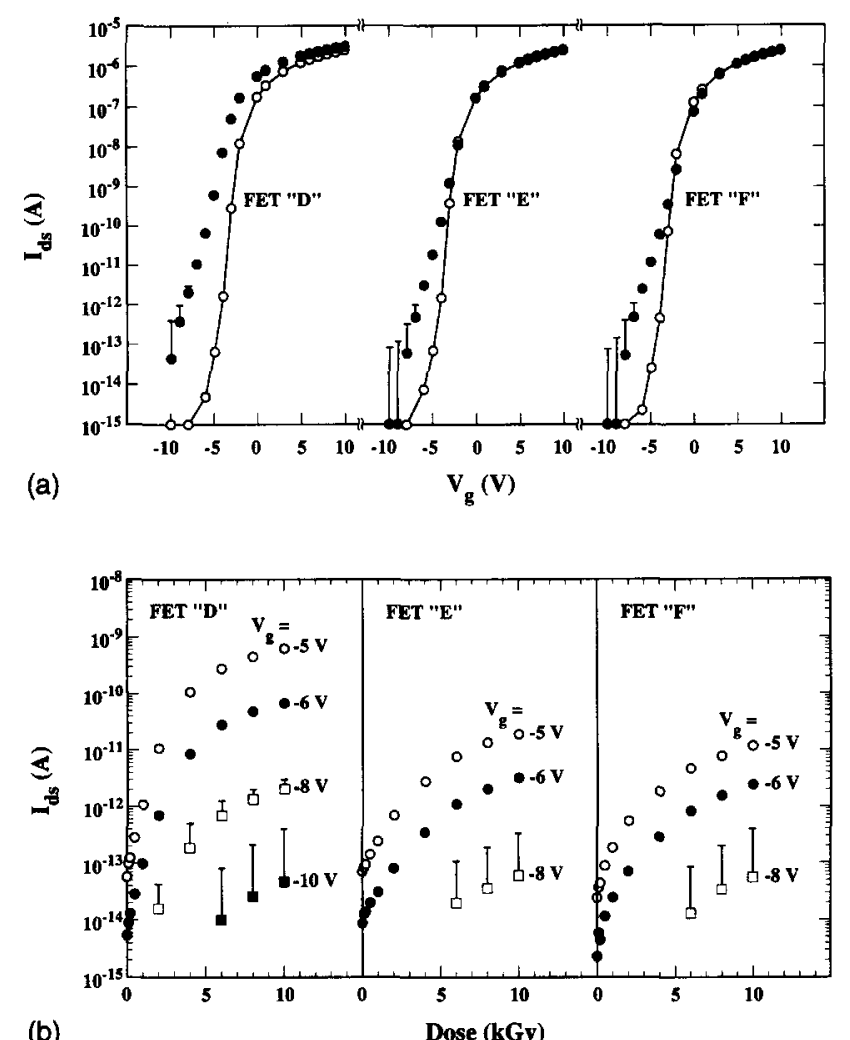

(b)

Dose (kGy)

FIG. 6. (a) Transfer characteristics for the three irradiation \#2 FETs measured prior to irradiation (open circles) and after $\sim 1 \times 10^{4}$ Gy (closed circles). (b) The radiation response of the three irradiation \#2 FETs for $V_{\mathrm{ds}}=-4 \mathrm{~V}$ and various $V_{g}$ settings. Transfer characteristics for $V_{\mathrm{ds}}=-2 \mathrm{~V}$ were also measured (see text for details).

$-2 \mathrm{~V}$ along the $V_{g}$ axis. For $V_{g}=-10 \mathrm{~V}, I_{\mathrm{ds}}$ values for $V_{\mathrm{ds}}=-2 \mathrm{~V}$ were bounded by those at $V_{\mathrm{ds}}=-4 \mathrm{~V}$, and for $V_{g} \geqslant 5 \mathrm{~V}, I_{\mathrm{ds}}$ values obeyed Eq. (1).

\section{Noise characteristics}

a. Positive $V_{g}$. In a previous study, ${ }^{18}$ it was shown that the magnitude of $S_{F}$ and $S_{T}$ can be predicted with reasonable accuracy using theoretical expressions ${ }^{26,27}$ developed for crystalline devices:

$$
\begin{aligned}
& S_{F}=\frac{\alpha q \mu^{2} \Gamma(W / L)^{2}}{(W L) f}\left(V_{g}-V_{T}-\frac{V_{\mathrm{ds}}}{2}\right) V_{\mathrm{ds}}^{2}, \\
& S_{T}=\beta 4 k T \mu \Gamma(W / L)\left(V_{g}-V_{T}\right) .
\end{aligned}
$$

For these expressions, $\alpha$ is relatively constant, varying between $\sim 2 \times 10^{-3}$ and $1 \times 10^{-2}, q$ is the magnitude of the electron charge, $\beta$ is a number ranging from unity $\left(V_{\mathrm{ds}} \ll V_{g}-V_{T}\right)$ to two-thirds $\left(V_{\mathrm{ds}} \geqslant V_{g}-V_{T}\right), k$ is Boltzmann's constant, and $T$ is the absolute temperature.

Measurements of the flicker and thermal noise components as a function of radiation dose for irradiation \#1 were compared with the above expressions, incorporating the changing $\mu$ and $V_{T}$ values (see Fig. 4). For $S_{F}$, the spectral noise was well characterized by the function $\mathrm{FC}_{\text {on }} / f^{\gamma}$, where $\mathrm{FC}_{\text {on }}$ is a coefficient denoting the magnitude of the flicker noise component (with the FET conducting or "on") and $\gamma$

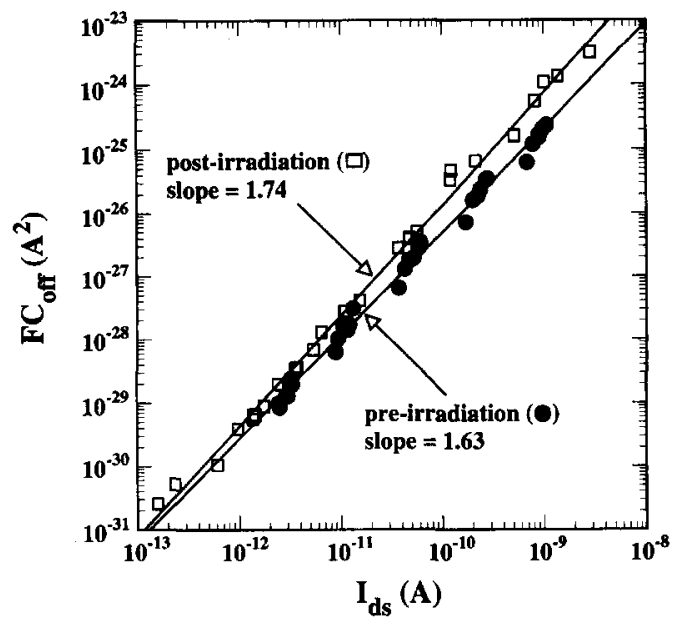

FIG. 7. $\mathrm{FC}_{\text {off }}$ values measured prior to and during the room-temperature annealing interval following irradiation \#1. The dependence of $\mathrm{FC}_{\mathrm{off}}$ on $I_{\mathrm{ds}}$ is only slightly altered by the $\sim 2 \times 10^{4}$ Gy irradiation.

describes the frequency dependence. Both $\mathrm{FC}_{\mathrm{on}}$ and $\gamma$ were determined from fits to the measured power spectra. Fits were performed to a maximum frequency of $\sim 10 \mathrm{~Hz}$. The value of $\alpha$ was then determined using Eq. (2) and the measured $\mathrm{FC}_{\mathrm{on}}$ value. Over the course of irradiation \#1, the value of $\gamma$ varied little and was equal to $1.07 \pm 0.02$. The value of $\alpha$ for the three irradiated FETs ranged from $\sim 4.6 \times 10^{-3}$ to $5.8 \times 10^{-3}$, in good agreement with previous results. ${ }^{18} \mathrm{Mea}-$ surements of the thermal noise component conformed well with theory, differing by a maximum of $5 \%$ from values predicted by Eq. (3).

For irradiation \#2, the value of $\gamma$ was not significantly affected by the radiation or the $V_{\mathrm{ds}}$ settings and was equal to $1.07 \pm 0.02$. Prior to the irradiation, $\alpha$ ranged from $\sim 4 \times 10^{-3}$ to $6 \times 10^{-3}$ for both $V_{\mathrm{ds}}$ settings. After the irradiation, $\alpha$ was again in this range except for FET $\mathrm{C}$, where $\alpha$ was $\sim 8.5 \times 10^{-3}$ for $V_{\mathrm{ds}}=4 \mathrm{~V}$. This deviation is small and is still within the range reported elsewhere. ${ }^{18}$ Thermal noise values before and after the irradiation differed from theory by a maximum of $\sim 5 \%$. These results for both irradiations \#1 and \#2 demonstrate that Eqs. (2) and (3) can be used independently of dose to determine $S_{F}$ and $S_{T}$, provided that the changes in $\mu$ and $V_{T}$ are taken into account.

b. Negative $V_{g}$. For $V_{g}=-5 \mathrm{~V}$ and various $V_{\mathrm{ds}}$, a noise component of the form $\mathrm{FC}_{\text {off }} f f^{\delta}$ was measured, where $\mathrm{FC}_{\text {off }}$ and $\delta$ were determined from fits to the measured power spectra. The average value of $\delta$ for the measurements before and after irradiation \#1 was $0.77 \pm 0.09$. $\mathrm{FC}_{\text {off }}$ depended on $I_{\mathrm{ds}}$ in a power-law fashion, as illustrated in Fig. 7. This power-law relationship is seen to be nearly the same both before and after the irradiation, where the slope of the "before" and "after" power law fits are $\sim 1.7$ and 1.6 , respectively. A difference between these data, not evident in Fig. 7, is that the $V_{\mathrm{ds}}$ values for a given $I_{\mathrm{ds}}$ were significantly different, up to $3 \mathrm{~V}$ greater for measurements performed after the irradiation. This is a result of the increase in $I_{\mathrm{ds}}$ with dose for a given $V_{\mathrm{ds}}$ value (see Fig. 5). 


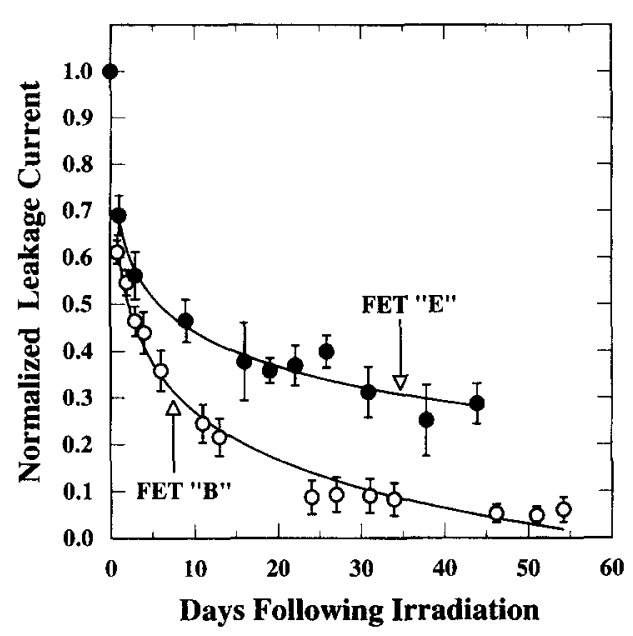

FIG. 8. Room-temperature annealing for FETs B and E. The leakage current was normalized to values measured immediately following the last irradiation interval, also referred to as the day 0 value. The lines through the data represent logarithmic fits. The behavior of FETs D and F were similar, respectively, to FETs $B$ and $E$ and are not shown for clarity.

\section{B. Annealing measurements}

\section{Room-temperature annealing}

Following both irradiations \#1 and \#2, the FET leakage current was observed to decrease with time following the final irradiation interval. This is shown in Fig. 8 for FETs B and $\mathrm{E}$. In this figure, the leakage current, normalized to the initial (day 0 ) value and averaged for the different voltage settings $\left(-10 \leqslant V_{g} \leqslant-3 \mathrm{~V}\right.$ and $V_{\mathrm{ds}}>V_{g}$ by $1 \mathrm{~V}$ or more), is plotted as a function of time following the respective irradiations. The behavior of $I_{\mathrm{ds}}$ for FETs B and E is such that $I_{\mathrm{ds}}$ decreased by $\sim 30 \%$ to $40 \%$ after the first day. Thereafter, $I_{\mathrm{ds}}$ decreases logarithmically with time. The room-temperature annealing behavior for FETs D and F was similar to that of FETs B and E, respectively. For FETs A and C, the leakage current decreased slowly with time following the irradiation but did not show a clear logarithmic dependence. $I_{\mathrm{ds}}$ measurements one day after the irradiation for FETs A and C were $\sim 10 \%$ and $50 \%$ lower, respectively, than the day 0 values and 50 days later were $\sim 5 \%$ to $20 \%$ of the day 0 values.

The mobility, threshold voltage, and linearity were also measured during the room-temperature annealing studies. The mobility remained constant or decreased slightly with a maximum decrease of $\sim 0.02 \mathrm{~cm}^{2} / \mathrm{Vs}$. The threshold voltage either remained stable or began to slowly relax back to the preirradiation values. The largest changes in $V_{T}$ occurred for FETs A, B, and D, which increased $\sim 0.3$ to $0.5 \mathrm{~V}$ from the day 0 values. For the remaining FETs, $V_{T}$ was stable, fluctuating a maximum of $\pm 0.1 \mathrm{~V}$ (relative to the day 0 values) over the $\sim 50$ day room-temperature annealing intervals. Linearity measurements performed after irradiation $\# 2$ demonstrated good agreement between $I_{\mathrm{ds}}$ and Eq. (1) $(\sim 4 \%)$ for $V_{g}=10 \mathrm{~V}$ and $-2 \leqslant V_{\mathrm{ds}} \leqslant 2 \mathrm{~V}$.

\section{Elevated-temperature annealing}

Following irradiation \#1 and the respective roomtemperature annealing measurements, 12 and 60 hour anneals at $\sim 45^{\circ} \mathrm{C}$ were carried out. After each of these anneals, the change in leakage current, $\mu$, and $V_{T}$ could not be differentiated from that expected due to room-temperature annealing. Subsequent to the $45^{\circ} \mathrm{C}$ anneals were several $2-4$ $\mathrm{h}$ anneals at $180^{\circ} \mathrm{C}$. After a cumulative anneal time of $12 \mathrm{~h}$ at $180^{\circ} \mathrm{C}$, the FET characteristics indicated nearly complete annealing: leakage current values for the irradiated FETs were within $\sim 30 \mathrm{fA}$ of the initial, preirradiation values; the mobility showed a small net increase $\left(\sim 0.04 \mathrm{~cm}^{2} / \mathrm{Vs}\right)$; and $V_{T}$ was $\sim 10 \%$ to $15 \%$ less than the preirradiation values. It is to note that the first $2 \mathrm{~h}$ anneal at $180^{\circ} \mathrm{C}$ had the dominant effect in changing the FET characteristics when compared to the subsequent $180^{\circ} \mathrm{C}$ anneals. (For $V_{g}=-5 \mathrm{~V}$ and $V_{\mathrm{ds}} \leqslant-2$ $\mathrm{V}$, the leakage current decreased by as much as a factor of $\sim 240$ following this first anneal with the subsequent anneals combining for a factor of $\sim 4$ to 5 decrease in leakage current.) In addition, the electrical properties $\left(\mu, V_{T}\right.$, and leakage current) for the three irradiated FETs were nearly the same after the first $180^{\circ} \mathrm{C}$ anneal, and the $\mu$ and $V_{T}$ values changed little $(\sim 8 \%)$ for the succeeding anneals.

Following the room-temperature annealing measurements associated with irradiation \#2, FET transfer characteristic measurements after a $2 \mathrm{~h}, 200^{\circ} \mathrm{C}$ anneal indicated complete annealing of the FETs to their respective preirradiation states.

\section{DISCUSSION}

\section{A. Radiation damage effects}

\section{Density of states of amorphous sillicon}

Before discussing possible radiation damage mechanisms, it is helpful to consider the energy distribution of electronic states, or the density of states, in the $a-\mathrm{Si}: \mathrm{H}$ layer near the interface with the bottom dielectric layer. This is illustrated in Fig. 9. It is observed that the density of states decreases exponentially from the valence and conduction band edges $\left(E_{v}\right.$ and $E_{c}$ ) toward the center of the gap. (The gap is defined by the range of energies lying between the valence and conduction band energies.) Because of the exponential falloff, the number of states near the center of the gap, or the number of deep states, is approximately 5-6 orders of magnitude less than those states near $E_{v}$ and $E_{c}$. In general, the states near $E_{v}$ and $E_{c}$, also known as the tail states, correspond to deviations in interatomic spacing. Structural defects resulting in broken or dangling bonds cause additional states throughout the gap (and can result in localized peaks, not shown in the figure, in the gap region). The distribution of states differs from crystalline silicon in that there are virtually no states (or orders of magnitude fewer than for $a-\mathrm{Si}: \mathrm{H}$ ) in the gap for crystalline silicon. This difference is largely respon- 


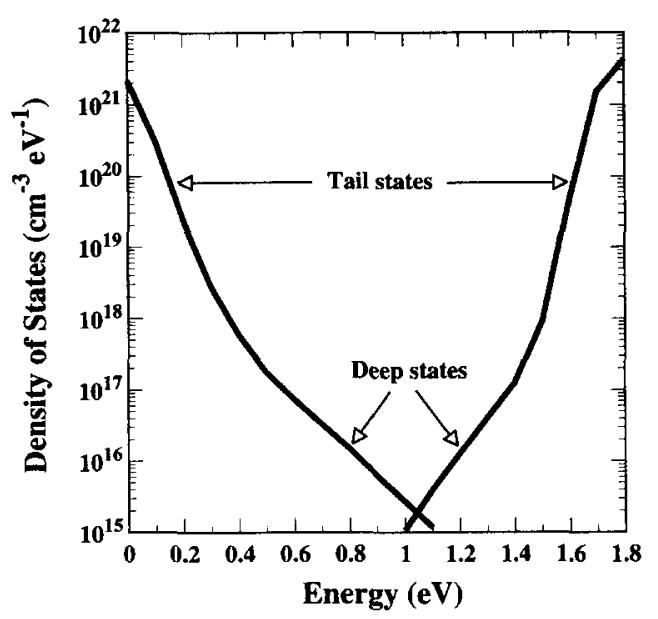

FIG. 9. Density of states for $a-S i: H$. The valence and conduction band edges are at 0 and $\sim 1.7 \mathrm{eV}$, respectively. The two curves correspond to different types of electronic states. The curve beginning at $0 \mathrm{eV}$ is for donorlike states while the curve ending at $1.8 \mathrm{eV}$ is for acceptorlike states. [Adapted from M. Shur et al., J. Appl. Phys. 66, 3371-3380 (1989).]

sible for the different electrical properties of crystalline and amorphous silicon FETs.

\section{Radiation damage mechanisms}

The results of irradiations \#1 and \#2 can largely be explained by considering the following two radiation damage mechanisms. The first is the creation of fixed positive charges, or holes, in the bottom dielectric layer (first suggested by French et al. ${ }^{16}$ as a damage mechanism for $a-\mathrm{Si}: \mathrm{H}$ FETs), and the second involves the creation of defect states in the $a-\mathrm{Si}: \mathrm{H}$ layer near the interface with the bottom dielectric layer. (Creation of holes in the top dielectric or passivation layer and of defects near the interface of the top dielectric and $a-\mathrm{Si}: \mathrm{H}$ layers may also be damage mechanisms with similar effects.) For hole creation in the bottom dielectric, incident radiation initially creates electron-hole pairs. The more mobile electrons are more readily swept out by the electric field (due to the bias applied during the irradiation), leaving a net excess of holes, which then act as fixed (or nearly so) positive charge centers. The holes will primarily act to decrease $V_{T}$, as their presence will reduce the positive voltage required to turn the FET on. The creation of defect states in the $a-\mathrm{Si}: \mathrm{H}$ layer near the bottom dielectric could arise from many interactions with the incident radiation such as the breaking of weak $\mathrm{Si}-\mathrm{Si}$ bonds or $\mathrm{Si}-\mathrm{H}$ bonds. Whatever the mechanism, it is expected that these additional defects will act as trapping centers with energies distributed across the gap (see Fig. 9). Because of these additional states, $V_{T}$ will increase (counteracting the effect of hole creation in the bottom dielectric layer) as a result of the larger $V_{g}$ necessary to compensate for the filling of the additional, radiation-induced trapping centers. It is also expected that these additional traps will decrease the subthreshold slope (in units of decades $/ V$ ), resulting in a less-sharp transition region.

\section{Drain-source current characteristics}

The two damage mechanisms discussed above can largely explain the effect of radiation on the drain-source current characteristics. This is discussed below.

a. Mobility. The mobility of the FETs (Sec. III A 1 a) was not significantly altered for either irradiation \#1 or \#2 and their respective annealing studies. Since the mobility is largely affected by the tail states, this result suggests that the radiation did not cause a significant increase (as compared to the initial distribution) in the number of these states.

b. Linearity. As with the mobility, the FET linearity (Sec. III A $l b$ ) was relatively unaffected for both irradiations and during the annealing studies. This result is evidence that the radiation did not cause significant changes in the $n$-doped layers, which are responsible for an ohmic connection between the drain and source contacts and the $a-\mathrm{Si}: \mathrm{H}$ layer.

c. Threshold voltage. For both irradiations \#1 and \#2 (Sec. III A 1 a, Fig. 4), $V_{T}$ for each of the FETs decreased initially and then remained constant or slightly increased with dose. Such behavior is consistent with the view that creation of holes in the dielectric (rather than state creation in the $a$-Si:H layer) is the initial dominant effect, causing a net decrease in $V_{T}$. As the dose level increases, state creation in the $a-\mathrm{Si}: \mathrm{H}$ layer may play a more significant role, resulting in $V_{T}$ flattening or slightly increasing with dose.

The behavior of $V_{T}$ with radiation dose was largely governed by the bias voltage applied to the FETs during the various irradiation intervals (see Table I). For irradiation \#1, FETs with smaller differences between $V_{g}$ and $V_{\mathrm{ds}}$ showed larger net $V_{T}$ shifts. This is probably a result of the lower electric field between the gate and drain contacts which is less effective for sweeping out holes that are created in the bottom dielectric layer or in moving these holes a further distance from the $a-\mathrm{Si}: \mathrm{H}$ layer (where they have less influence on the FET properties). For irradiation \#2, FETs E and $F$ showed nearly the same response (offset by different initial values), and $V_{T}$ showed the smallest initial decrease with dose (when compared with FETs from both irradiations) after which $V_{T}$ slowly increased with dose. This suggests that a gate voltage of $-10 \mathrm{~V}$ (as compared to $V_{g}=-5 \mathrm{~V}$ for the other FETs) was more effective in suppressing the creation of holes in the bottom dielectric layer and that this suppression was nearly independent of $V_{\mathrm{ds}}$ for more negative $V_{g}$ $\left(V_{g}=-10 \mathrm{~V}\right)$.

d. Leakage current and transfer characteristic. As shown in Fig. 5, the leakage current for $V_{g}=-5 \mathrm{~V}$ and $V_{\mathrm{ds}} \leqslant 0 \mathrm{~V}$ dramatically increased with dose. This behavior is consistent with the transfer characteristic behavior (Sec. III A $1 d$, Fig. 6) at $V_{g}=-5 \mathrm{~V}$ and will be discussed in conjunction with this data.

As with the $V_{T}$ data, the transfer characteristic behavior can be viewed as resulting from the creation of holes in the dielectric layer and from state creation in the $a-\mathrm{Si}: \mathrm{H}$ layer. Creation of holes in the dielectric will cause a shift in the transfer characteristic in the direction of the $V_{T}$ change, and state creation in the $a-\mathrm{Si}: \mathrm{H}$ layer will cause a decrease in the 
subthreshold slope. The combination of these two effects will result in a larger leakage current value for a given $V_{g}$, as is evident in Fig. 6. This increase in leakage current becomes more pronounced for $V_{g}$ near the transition region from the nonconducting to conducting states. This is especially evident for the leakage current measurements at $V_{g}=-5 \mathrm{~V}$ and $V_{\mathrm{ds}}=-4 \mathrm{~V}$. For more negative $V_{g}$ which are initially further from the transition region (such as $V_{g}=-10 \mathrm{~V}, V_{\mathrm{ds}}=-4 \mathrm{~V}$ ), the effect of the radiation is not nearly as significant. (This is also evident for leakage current measurements at $V_{g}=-5 \mathrm{~V}$ and $V_{\mathrm{ds}}=+4$ V.)

The degree to which leakage current was affected by the radiation also depended on the bias voltage that was applied during the irradiation intervals. For irradiation \#1, a smaller difference between $V_{g}$ and $V_{\mathrm{ds}}$ resulted in a larger rise in leakage current. This dependence is analogous to the corresponding $V_{T}$ behavior. As the difference between $V_{g}$ and $V_{\mathrm{ds}}$ decreases, the shift in $V_{T}$ and the corresponding characteristic is larger, resulting in a larger increase in the leakage current. For irradiation \#2, leakage current for the two FETs biased at $V_{g}=-10 \mathrm{~V}$ (FET E and F) showed nearly the same behavior and showed a smaller increase with dose than FET $\mathrm{D}$, which had a less negative $V_{g}$ applied $\left(V_{g}=-5 \mathrm{~V}\right)$. This suggests that for more negative $V_{g}$, creation of holes and states in the gap are suppressed and less dependent on $V_{\mathrm{ds}}$.

\section{B. Implications for $x$-ray imaging}

No significant effect upon the mobility, linearity, and noise properties of the FETs was observed up to the maximum cumulative dose measured $\left(\sim 2 \times 10^{4}\right.$ Gy). However, the threshold voltage and the transfer characteristic of the FET both demonstrated changing behavior with increasing dose. It is useful to view the significance of these changes in the context of the expected lifetime dose to an imager for a given application.

\section{Radiation effects on array FETs for general diagnostic applications}

While routine use of an imager in a radiotherapy environment can be expected to deliver doses of up to $\sim 2 \times 10^{4}$ Gy per year, the lifetime dose for diagnostic equipment used 250 days per year over 7 years can be several orders of magnitude less. Based on an estimate of 100 images per day, a general chest radiography ( $\sim 0.025 \mathrm{cGy}$ per image) and mammography ( $\sim 1$ cGy per image) unit would receive lifetime doses of $\sim 44$ Gy and $\sim 1800$ Gy respectively. (For mammography, the calculation assumes: 25 patients/day $\times 4$ images/patient, giving approximately 100 images per day.) These estimates account for the effect of the unattenuated beam. For a fluoroscopic unit, based on an average of 102 $\mu \mathrm{R}$ per second ${ }^{28}$ with the unit delivering $\mathrm{x}$ rays $20 \%$ of the time over a $10 \mathrm{~h}$ day, the estimated lifetime dose is $\sim 13 \mathrm{~Gy}$ (assuming $1 \mathrm{R}$ corresponds to $\sim 1 \mathrm{cGy}$ ). However, if the fluoroscopic unit were operated so that the imager was exposed to the unattenuated beam a significant portion of the time, this estimate could increase by up to perhaps a factor of 100. These lifetime dose estimates for diagnostic imagers are probably conservative overestimates of the dose delivered to the $a$-Si:H FETs as they ignore the attenuation of primary and scattered radiation in the overlying $x$-ray converter.

The first irradiation steps of 100 and $200 \mathrm{~Gy}$ were larger than the estimated lifetime doses of a chest radiography imager as well as fluoroscopic imagers rarely $(<\sim 10 \%)$ detecting the unattenuated beam. The relatively small effects on the FETs at these dose levels (measured and interpolated changes of less than $0.1 \mathrm{~V}$ in threshold voltage, and leakage current magnitudes less than or equal to $\sim 100 \mathrm{fA}$ ) are unlikely to affect the performance of an imager. Moreover, given that the 100 and $200 \mathrm{~Gy}$ were delivered in less than an hour and given the restorative effect of room-temperature annealing following the irradiations, one might expect little or no net change in the properties of the FETs at the dose rates to be expected in routine clinical use for these applications. In the case of mammographic imaging and fluoroscopic imaging (with the detector frequently exposed to the unattenuated beam), the effect of room-temperature annealing may be insufficient to cancel the effect of the radiation on the FETs and the considerations of the next section may apply.

\section{Radiation effects on array FETs for high dose applications}

The large changes observed in threshold voltage and transfer characteristic of the FETs at dose levels commensurate with those of megavoltage imaging can be expected to lead to a measurable effect upon the operation of the array. Most significantly, an increase in leakage current at negative gate voltage would affect, to varying degrees, the effective signal capacity of the photodiode sensor, the noise of the photodiode, and the noise integrated by the external preamplifier connected to a given column of pixels. It should be noted, however, that the effects may be somewhat offset by room-temperature annealing since the present data was acquired at an average dose rate approximately 30 times higher than that expected clinically (12 days vs a year for the $\sim 2 \times 10^{4}$ Gy irradiation).

a. Effect on pixel signal. For a given negative gate voltage, the leakage current was generally observed to increase with dose. This effect will increase the amount of charge subtracted from that integrated in the photodiode (due to the incident $x$ rays and the photodiode leakage current). The importance of this effect depends upon the design of the pixel. For example, assume a preirradiated FET leakage current of $1 \mathrm{fA}$. In addition, the capacitance per unit area for $1 \mu \mathrm{m}$ thick photodiode sensors is $\sim 100 \mathrm{pF} / \mathrm{mm}^{2}$. For a $450 \mu \mathrm{m}$ pixel-to-pixel pitch array ${ }^{3}$ with a geometric fill factor of $67 \%$, a photodiode reverse bias of $-4 \mathrm{~V}$ and operated at a frame rate of $1 \mathrm{fps}$, the percentage of the charge subtracted by the FET leakage current, as compared to photodiode saturation, increases from $0.002 \%$ (preirradiation) to $0.02 \%$, $0.2 \%$, and $2 \%$ for 1,2 , and 3 orders of magnitude increase in $I_{\mathrm{ds}}$. For a $127 \mu \mathrm{m}$ pitch array with a geometric fill factor of $42 \%$ (Ref. 29) and a frame rate of $1 \mathrm{fps}$, the percentage increases from $0.04 \%$ (preirradiation) to $0.4 \%, 4 \%$, and $40 \%$ for 1,2 , and 3 order of magnitude increases. (Note that the 
percentage of charge capacity subtracted decreases as the frame rate increases.) Thus, the effect of the radiation becomes significant when the leakage current is sufficiently increased.

b. Effect on pixel noise. For an array pixel, the noise contribution from the FET (during the time that the FET is in the nonconducting state), will increase with leakage current. As illustrated in Fig. 7, the flicker noise component of the noise-power-spectral density depends on $I_{\mathrm{ds}}$ in a power-law fashion and a shot noise component is anticipated to equal $2 q\left|I_{\mathrm{ds}}\right|$. Therefore, increases in the leakage current with cumulative radiation dose may result in a degradation of the signal to noise ratio of an individual array pixel.

While in the conducting state, the FET thermal noise is anticipated to be the dominant noise source, contributing an amount proportional to ${ } \mathrm{kTC}$ coulombs (where $\mathrm{C}$ is the sensor capacitance), ${ }^{1,30,31}$ provided the FET can be modeled as a resistor. Measurements of the FET in the conducting state for both irradiations \#1 and \#2 $\left(\mu, V_{T}\right.$, linearity, and noise) indicate that the FET behaves like a resistor and, therefore, it is not expected that the noise contribution due to FET thermal noise will vary with increasing dose.

\section{Strategies to compensate for the effect of radiation on array FETs}

While room-temperature annealing may largely reduce or even eliminate the effects of radiation on the FETs for low dose applications, it is expected that this will be insufficient to compensate for effects at higher doses. In this event, two strategies are possible. The elevated-temperature annealing measurements suggest that the FETs may be returned to their preirradiated state by means of a $2 \mathrm{~h}$ anneal at $\sim 200^{\circ} \mathrm{C}$. However, this procedure could prove impractical in a clinical setting for a variety of technical and/or logistical reasons. For example, it would probably be necessary to separate the array from the peripheral electronics in order to prevent damage to the electronics during a $200^{\circ} \mathrm{C}$ anneal. This may not be practical in a clinical setting as it would require a quick and reliable method for severing and later reestablishing electrical contact with the array-a difficult task considering the numerous array contact pads and the large area spanned by a typical array.

A second strategy would capitalize upon the observation that increases in leakage current were significantly less for gate voltages further removed from the subthreshold region (i.e., more negative gate voltages). The selection of the value of the negative gate voltage is governed by a number of considerations. It is desired that while in the "off" state, the FETs be as nonconducting as possible so as to minimize the leakage of current through the FET. This consideration would favor operation of the FETs at the most negative values of $V_{g}$ (e.g., $-8 \mathrm{~V}$ or beyond-see Fig. 2). However, the amount of charge injected into the photodiodes and the preamplifiers when the gate voltage is switched (the "switching transient") increases with the difference between "off" (i.e., negative) and "on" (i.e., positive) gate voltage. As this transient reduces the available signal capacity of the photodiode and can act as a source of noise, it is generally desirable to keep this difference as small as possible. For these reasons, a possible strategy would be to initially operate the arrays at a gate voltage giving a reasonable leakage (e.g., $-5 \mathrm{~V}$ ) prior to irradiation, and gradually shift the value of $V_{g}$ to more negative bias as the effects of cumulative dose accrue.

\section{SUMMARY}

The noise and drain-source current characteristics of $a-\mathrm{Si}: \mathrm{H}$ field-effect transistors were measured as a function of cumulative dose using a ${ }^{60} \mathrm{Co}$ source. Studies examining the effects of large cumulative doses (up to $\sim 2 \times 10^{4} \mathrm{~Gy}$ ) delivered at relatively high dose rates (as compared to medical imaging applications) were performed. Room-temperature and elevated-temperature annealing studies were also carried out. Neither the noise characteristics nor the mobility were significantly altered by the radiation. The net change in the threshold voltage was modest, $\sim 1.8 \mathrm{~V}$ maximum. The most significant effect of the radiation was a shift in the FET transfer characteristic toward more negative gate voltages and/or a decrease in the transfer characteristic subthreshold slope, resulting in increases in the FET leakage current. Such increases were more pronounced for gate voltages nearer the subthreshold region and were reduced by biasing the FET at more negative gate voltages while being irradiated. Roomtemperature annealing of the FETs resulted in initial leakage current decreases of $10 \%$ to $50 \%$ one day following the irradiation studies and thereafter the leakage current decreased logarithmically with time. Elevated-temperature anneals for $2 \mathrm{~h}$ at $200^{\circ} \mathrm{C}$ restored FET transfer characteristics to their preirradiation values. For imaging applications in chest radiography and fluoroscopy (with infrequent exposure to the direct beam), where the lifetime dose to a detector is anticipated to be less than $\sim 100 \mathrm{~Gy}$, the results of the irradiation studies indicated that $a$-Si:H FETs are sufficiently resistant to radiation damage. For higher-dose applications, such as mammography, fluoroscopy (when direct exposure to the radiation is frequent), and particularly radiotherapy, the results indicate that it may be necessary to either periodically anneal the arrays or, more practically, to operate the arrays with a more negative gate voltage as the cumulative dose increases. Further investigations into the underlying causes of these radiation-induced effects, including the effects of multiple irradiation and anneal cycles, are underway and possible changes to the structure of the FETs will be studied.

\section{ACKNOWLEDGMENTS}

We wish to thank Jeffrey H. Siewerdsen, Kyung-Wook Jee, and Donald L. Brooks for their assistance in the acquisition of the irradiation data. In addition, we thank Robert A. Street and Richard L. Weisfield of Xerox, PARC, for supplying the devices used in this study and for valuable discussions. J.H.S. is also acknowledged for helpful comments on the manuscript. This work was supported by National Institutes of Health Grant No. 2-R01-CA-51397. 
${ }^{1}$ L. E. Antonuk, J. M. Boudry, Y. El-Mohri, W. Huang, J. H. Siewerdsen, J. Yorkston, and R. A. Street, "Large area, flat-panel, amorphous silicon imagers," SPIE 2432 (Medical Imaging 1995: Physics of Medical Imaging), 216-227 (1995).

${ }^{2}$ R. A. Street, S. Nelson, L. E. Antonuk, and V. Perez-Mendez, "Amorphous silicon sensor arrays for radiation imaging," Mat. Res. Soc. Proc. 192, $441-452$ (1990)

${ }^{3}$ L. E. Antonuk, J. Boudry, W. Huang, D. L. McShan, E. J. Morton, J. Yorkston, M. J. Longo, and R. A. Street, "Demonstration of megavoltage and diagnostic $\mathrm{x}$-ray imaging with hydrogenated amorphous silicon arrays," Med. Phys. 19, 1455-1466 (1992).

${ }^{4}$ K. Kobayashi, S. Makida, Y. Sato, and T. Hamano, "640×400 pixels $a$-Si:H TFT driven 2-dimensional image sensor,' SPIE 1900 (Electronic Imaging: Charge-coupled Devices and Solid State Optical Sensors III), 40-46 (1993)

${ }^{5}$ M. J. Powell, I. D. French, J. R. Hughes, N. C. Bird, O. S. Davies, C. Glasse, and J. E. Curran, "Amorphous silicon image sensor arrays," Mat. Res. Soc. Proc. 258, 1127-1137 (1992).

${ }^{6}$ M. Yamaguchi, Y. Kaneko, and K. Tsutsui, "Two-dimensional contacttype image sensor using amorphous silicon photo-transistor," Jpn. J. Appl. Phys. 32, 458-461 (1993).

${ }^{7}$ D. L. Lee, L. K. Cheung, and L. S. Jeromin, "A new digital detector for projection radiography," SPIE 2432 (Medical Imaging 1995: Physics of Medical Imaging), 237-249 (1995).

${ }^{8}$ C. van Berkel, N. C. Bird, C. J. Curling, and I. D. French, “2D image sensing arrays with nip diodes," Mat. Res. Soc. Proc. 297, 939-944 (1993).

${ }^{9}$ K. Yamamoto, K. Sai, Y. Ohta, H. Mimura, and K. Kitamura, "Amorphous silicon 2-dimensional image sensor," Technical Digest of the 8th Sensor Symposium, 55-58 (1989).

${ }^{10} \mathrm{~T}$. Graeve, W. Huang, S. M. Alexander, and Y. Li, "Amorphous silicon image sensor for x-ray applications," SPIE 2415 (Electronic Imaging: Charge-coupled Devices and Solid State Optical Sensors V), 177-181 (1995).

${ }^{11}$ G. Cho, J. S. Drewery, W. S. Hong, T. Jing, H. Lee, S. N. Kaplan, A. Mireshghi, V. Perez-Mendez, and D. Wildermuth, "Utilization of $a-\mathrm{Si}: \mathrm{H}$ switching diodes for signal readout from $a-\mathrm{Si}: \mathrm{H}$ pixel detectors," Mat. Res. Soc. Symp. Proc. 297, 969-974 (1993).

${ }^{12}$ L. E. Antonuk, J. Yorkston, W. Huang, J. Boudry, E. J. Morton, and R. A. Street, "Large area, flat-panel $a-\mathrm{Si}: \mathrm{H}$ arrays for $\mathrm{X}$-ray imaging," SPIE 1896 (Medical Imaging 1993: Physics of Medical Imaging), 18-29 (1993).

${ }^{13}$ L. E. Antonuk, J. Yorkston, W. Huang, J. H. Siewerdsen, J. M. Boudry, Y. El-Mohri, and M. V. Marx, "A real-time, flat-panel, amorphous silicon, digital X-ray imager,' Radiographics 15, 993-1000 (1995).

${ }^{14} \mathrm{~J}$. M. Boudry and L. E. Antonuk, "Radiation damage of amorphous silicon photodiode sensors,” IEEE Trans. Nucl. Sci. 41, 703-707 (1994).

${ }^{15}$ L. E. Antonuk, J. Boudry, J. Yorkston, C. F. Wild, M. J. Longo, and R. A.
Street, "Radiation damage studies of amorphous-silicon photodiode sensors for applications in radiotherapy $x$-ray imaging," Nucl. Instr. Meth. A 299, 143-146 (1990).

${ }^{16}$ I. D. French, A. J. Snell, P. G. LeComber, and J. H. Stephen, "The effect of $\gamma$-irradiation on amorphous silicon field effect transistors," Appl. Phys. A 31, 19-22 (1983).

${ }^{17}$ G. Cho, M. Conti, J. S. Drewery, I. Fujieda, S. N. Kaplan, V. PerezMendez, and S. Qureshi, "Assessment of TFT amplifiers for $a-\mathrm{Si}: \mathrm{H}$ pixel particle detectors," IEEE Trans. Nucl. Sci. 37, 1142-1148 (1990).

${ }^{18} \mathrm{~J}$. M. Boudry and L. E. Antonuk, "Current-noise-power spectra of amorphous silicon thin-film transistors,' J. Appl. Phys. 76, 2529-2534 (1994).

${ }^{19}$ R. A. Street, Hydrogenated Amorphous Silicon (Cambridge University, Cambridge, 1991), Chap. 10.

${ }^{20}$ M. J. Powell, "The physics of amorphous-silicon thin-film transistors," IEEE Trans. Elec. Dev. 36, 2753-2763 (1989).

${ }^{21} \mathrm{C}$. van Berkel, in Amorphous and Microcrystalline Semiconductor Devices: Materials and Device Physics, edited by J. Kanicki (Artech House, Norwood, MA, 1992), Chap. 8.

${ }^{22}$ S. R. Hofstein, in Field-Effect Transistors, edited by J. T. Wallmark and H. Johnson (Prentice-Hall, Englewood Cliffs, NJ, 1966), Chap. 5.

${ }^{23}$ Task Group 21, Radiation Therapy Committee AAPM, "A protocol for the determination of absorbed dose from high-energy photon and electron beams," Med. Phys. 10, 741-771 (1983).

${ }^{24}$ M. J. Powell, C. van Berkel, I. D. French, and D. H. Nicholls, "Bias dependence of instability mechanisms in amorphous silicon thin-film transistors," Appl. Phys. Lett. 51, 1242-1244 (1987).

${ }^{25}$ J. Kanicki, C. Godet, and A. V. Gelatos, "Bias stress induced instabilities in amorphous silicon nitride/crystalline silicon and amorphous silicon nitride/amorphous silicon structures,' Mat. Res. Soc. Symp. Proc. 219, 45-50 (1991).

${ }^{26}$ A. van der Ziel, "Flicker noise in electronic devices," Adv. Electron. Electron Phys. 49, 225-297 (1979).

${ }^{27}$ A. G. Jordan and N. E. Jordan, "Theory of noise in metal oxide semiconductor devices," IEEE Trans. Electron Devices ED-12, 148-156 (1965).

${ }^{28}$ Task Group 11, "A survey of fluoroscopic exposure rates: AAPM Task Group No. 11 Report,” Med. Phys. 20, 789-794 (1993).

${ }^{29}$ L. E. Antonuk, Y. El-Mohri, W. Huang, J. Siewerdsen, J. Yorkston, and R. A. Street, "A large area, high-resolution $a-\mathrm{Si}: \mathrm{H}$ array for X-ray imaging," Mat. Res. Soc. Symp. Proc. 336, 855-860 (1994).

${ }^{30}$ U. Schiebel, N. Conrads, N. Jung, M. Weibrecht, H. Wieczorek, T. Zaengel, M. J. Powell, I. D. French, and C. Glasse, "Fluoroscopic x-ray imaging with amorphous silicon thin-film arrays," SPIE 2163 (Medical Imaging 1994: Physics of Medical Imaging), 129-140 (1994).

${ }^{31}$ I. Fujieda, R. A. Street, R. L. Weisfield, S. Nelson, P. Nylen, V. PerezMendez, and G. Cho, "High sensitivity readout of 2D a-Si image sensors,” Jpn. J. Appl, Phys. 32, 198-204 (1993). 